\title{
Efficacy of an Abduction Brace in Preventing Initial Dislocation in the Early Postoperative Period after Primary Total Hip Arthroplasty
}

\author{
Yoshinori Ishii ${ }^{1}$, Hideo Noguchi ${ }^{1}$, Mitsuhiro Takeda ${ }^{1}$, Junko Sato ${ }^{1}$, Yoichiro Domae ${ }^{2}$ \\ ${ }^{1}$ Ishii Orthopaedic and Rehabilitation Clinic, Saitama, Japan \\ ${ }^{2}$ Department of Orthopaedic Surgery, Shibata Prefectural Hospital, Niigata, Japan \\ Email: ishii@sakitama.or.jp
}

Received November 27, 2011; revised January 15, 2012; accepted January 28, 2012

\begin{abstract}
We hypothesized that a hip brace may prevent the initial dislocation in the early postoperative period after total hip arthroplasty (THA). We performed a prospective evaluation of the efficacy of a brace in preventing dislocation in 54 primary THAs in 43 patients. All of the patients wore braces for 3 weeks postoperatively. The occurrence of dislocation was evaluated six months postoperatively. There were no cases of dislocation while wearing a brace, while two posterior dislocations occurred 8 and 12 days postoperatively while picking something up from the floor without a brace in so-called provocative positions. The results of this study suggest that a hip brace helps patients to recognize careless provocative positions and prevents the initial hip dislocation in this period instead of usual postoperative management for an average of 6 weeks after discharge, such as a high toilet seat, restricted hip flexion in the activities of daily living, use of a reacher or grabber, an abduction pillow, and a high chair.
\end{abstract}

Keywords: Hip Brace; Dislocation; Total Hip Arthroplasty

\section{Introduction}

Postoperative dislocation remains a common major complication after total hip arthroplasty (THA) with an overall incidence of $2 \%$ to $3 \%$, but approaching less than $1 \%$ after primary THA [1-9]. Khatod et al. [10] reported dislocation rates of $1.7 \%$ for primary THA and $5.1 \%$ for revision procedures using contemporary techniques and implants with a community-based registry. Multiple factors have been discussed from patient and surgical perspectives in many reports of large series $[2,3,8,11,12]$. Patient factors include neuromuscular and cognitive disorders, patient non-compliance, and previous hip surgery $[8,13,14]$. Surgical considerations include the approach [9,15], soft tissue tension [5], component positioning [11, 16-18], impingement [19], femoral head size [3,20], acetabular liner profile [4], and surgeon experience [6]. The average time to the initial dislocation after a primary procedure is relatively short $[3,8,9,21]$. Recently, THA has become day surgery with the introduction of minimally invasive surgical methods [22]. Biedermann et al. [11] reported that most dislocations (119/137, 78\%) occurred within 12 weeks of surgery in 4784 THAs, and most occurred within 3 weeks. In the Medicare population, there was a 3.9\% dislocation rate in the first 6 months after primary THA [12]. The incidence of dislo- cation is highest immediately after THA, but it continues to be elevated throughout the first three postoperative months [12]. Therefore, it is important to decrease hip dislocation in the early postoperative period. This study was prospectively performed to evaluate the efficacy of bracing at preventing initial dislocations after primary THA during the early postoperative period.

\section{Patients and Methods}

We performed 54 primary THAs in 43 patients (47 hips in 38 women and 7 hips in 5 men) in our clinic. The average age was 62 years (range 44 to 83 ) and average height and weight were $154 \mathrm{~cm}$ and $56 \mathrm{~kg}$, respectively. The average body mass index (BMI) was 24 (range 19 to 32) (Table 1). Preoperative diagnosis was osteoarthritis in all cases.

Table 1. Patient characteristics.

\begin{tabular}{cc}
\hline Number of patients & 43 Patients, 54 Hips \\
Mean age & $62 \pm 11$ years \\
Sex (Male:Female) & 5 (7 THA):Female 38 (47 THA) \\
Body height & $154 \pm 7 \mathrm{~cm}$ \\
Body weight & $56 \pm 9 \mathrm{~kg}$ \\
BMI & $24 \pm 3$ \\
Operated side & 25 left hips, 29 right hips \\
Surgical approach & 22 lateral, 14 posterior, 18 anterior \\
\hline
\end{tabular}


The surgical procedure was performed by three senior surgeons and oriented the acetabular component in a position of $35^{\circ}$ to $45^{\circ}$ of abduction in the coronal plane and $20^{\circ}$ of anteversion in the sagittal plane, whereas the femoral component was oriented at $15^{\circ}$ of anteversion. Meticulous soft tissue reconstruction was performed in all approaches to attain proper stability and to minimize dislocation.

All patients wore hip braces from just after surgery to 3 weeks postoperatively. Full weight-bearing was allowed as tolerated with a cane on the first postoperative day, under the supervision of a therapist, and exercise was allowed. All patients in this study were clearly instructed to avoid crossing their legs, hip flexion beyond $90^{\circ}$, and excessive rotation for at least 6 weeks postoperatively. Each patient had close follow-up for at least 6 months postoperatively. As the average hospital stay was $40 \pm 18$ days (range 9 to 99) and all patients received rehabilitation under supervision of a physical therapist between 3 to 4 times a week for 6 months, all of the dislocations were reported. The average hospital stay was much longer than in the US (3.5 [23] to 5.0 [12] days for primary THA). As the rehabilitation protocol and postoperative complaints did not differ remarkably, the difference in the US and Japan medical insurance systems likely influenced this result. The occurrence of an initial dislocation was evaluated at 6 months postoperatively to assess the efficacy of applying a hip brace in the early postoperative period.

The abduction brace used has a waistband that can be adjusted using hook-and-loop straps. The joint is an adjustable metal joint with both flexion and abduction stops that were deemed optimal by the surgeons at the level of the hip. Distal to the hip joint is an adjustable thigh cuff, also tightened with hook-and-loop straps. Braces were made individually, fitted to each patient, and applied in accordance with the manufacturer's instructions (TechnoBrace CC, Kazo, Saitama, Japan) (Figure 1).

The brace was worn continuously, except when bathing, for 3 weeks. The hip abduction brace costs approximately \$800. Patient demographics, surgical approach, and femoral head sizes are listed in Tables 1 and 2. Implant types are listed in Table 3.

Abduction and anteversion of the acetabular component were analyzed on available radiographs with Murray's view [24] at three months postoperatively. The parameters used to determine proper component position were described by Lewinnek et al. [18] as the "safe position," with the acetabular component in the range of $15^{\circ}$ $\pm 10^{\circ}$ anteversion and $40^{\circ} \pm 10^{\circ}$ abduction. Components outside these parameters were considered malpositioned. Acetabular component malposition was determined radiographically. The degree of component abduction was measured from the angle formed by a line drawn through

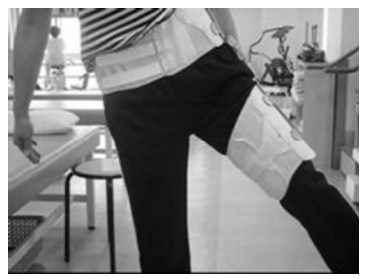

(a)

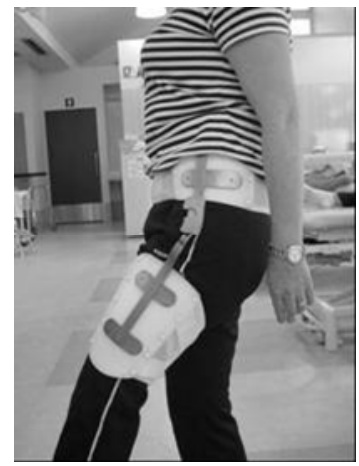

(c)

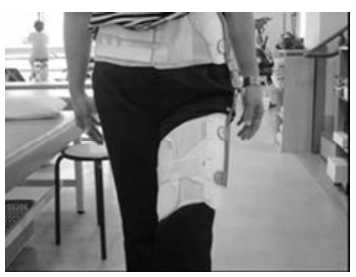

(b)

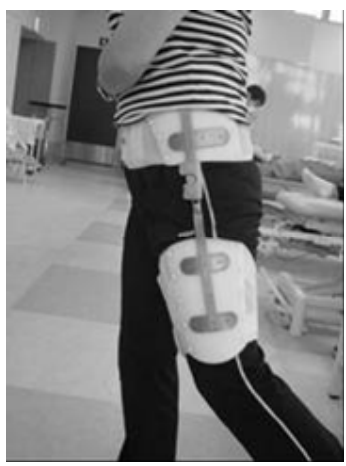

(d)
Figure 1 (a) Abduction; (b) Adduction; (c) Flexion; (d) Extension The brace allows the patient to abduct $10^{\circ}$ and flex $70^{\circ}$ maximally, while it stops adduction and extension.

Table 2. Component profile.

\begin{tabular}{cc}
\hline Component head size & Number \\
\hline $22 \mathrm{~mm}$ & 17 \\
$26 \mathrm{~mm}$ & 29 \\
$28 \mathrm{~mm}$ & 5 \\
$28 \mathrm{~mm}$ & 2 ceramic \\
$36 \mathrm{~mm}$ & 1 \\
Component cup size & \\
$46 \mathrm{~mm}$ & 3 \\
$48 \mathrm{~mm}$ & 12 \\
$50 \mathrm{~mm}$ & 20 \\
$52 \mathrm{~mm}$ & 10 \\
$54 \mathrm{~mm}$ & 5 \\
$56 \mathrm{~mm}$ & 4 \\
\hline
\end{tabular}

Table 3. Implant types.

\begin{tabular}{cc}
\hline Acetabular component & 32 \\
\hline Depuy AML plus & 3 \\
Depuy C-stem & 19 \\
Aesculap Bicontact N & \\
Femoral component & 32 \\
Depuy AML plus & 3 \\
Depuy C-stem & 19 \\
Aesculap Bicontact N
\end{tabular}


both acetabular teardrops and a line drawn tangential to the face of the acetabulum. Anteversion and retroversion were measured on the lateral radiograph as the angle formed by the line tangential to the face of the acetabular component and the line perpendicular to the horizontal plane, which determined the degree of anteversion.

\section{Results}

Although no dislocation occurred while wearing a brace for 6 months postoperatively, two hip posterior dislocations occurred 8 and 12 days postoperatively while picking something up from the floor carelessly without wearing the brace in a knee-in posture (the so-called provocative position; flexion $>90^{\circ}$, adduction, and internal rotation) while in hospital. In both cases, THA was performed using a posterior approach with femoral head sizes of 22 and $26 \mathrm{~mm}$, respectively. Judging from the radiographic evaluations, both dislocated cases were within the safe range based on the definition of Lewinnek et al. [18] The average abduction was $41^{\circ}$ (range $30^{\circ}$ to $50^{\circ}$ ) and the average anteversion was $11^{\circ}$ (range $2^{\circ}$ to $19^{\circ}$ ) (Table 4).

\section{Discussion}

As postoperative dislocation remains a common major complication after THA, many reports have discussed patient and surgical risk factors in large series [2,3,8, 11,12]. As surgical risk factors are related to the surgical approach, some authors recommend anterior [9] or direct lateral [15] approaches rather than a posterior approach, while others concluded that the posterior, posterolateral, and direct lateral approaches have equally excellent dislocation rates $(<1 \%)$ when the approach incorporates definitive posterior soft-tissue repair [5,7,25,26]. Postoperative soft-tissue tension (created by the joint capsule, short external rotators, and gluteal muscles) has been examined carefully as a factor in dislocation [19]. Therefore, the maintenance of the proper component positioning in the early postoperative period after meticulous soft tissue repair may be regarded as crucial, as most dislocations are reported to occur within 12 weeks of surgery, especially within 3 weeks [11].

As THA is approaching day surgery with the introducetion of minimally invasive surgical techniques [22], it is critical for patients who undergo THA to avoid provocative positions after discharge. Functional restrictions following THA are thought to reduce the prevalence of early postoperative dislocation, although Peak et al. [23]

Table 4. Radiographic evaluation.

\begin{tabular}{cc}
\hline X-p Abduction & X-p Anteversion \\
\hline $41^{\circ} \pm 4^{\circ}$ & $11^{\circ} \pm 4^{\circ}$ \\
\hline
\end{tabular}

reported that the removal of several patient restrictions did not increase the prevalence of dislocation following primary hip arthroplasty. Youm et al. [27] investigated issues such as postoperative rehabilitation and activity restriction in a survey of active members of the American Association of Hip and Knee Surgeons. Ninety percent of responders (300/336) included dislocation precautions as part of their patients' postoperative management for an average of 6 weeks after discharge, such as a high toilet seat, restricted hip flexion in the activities of daily living (ADL), use of a reacher or grabber, an abduction pillow, and a high chair. Applying an abduction brace after THA seems to help the patients recognize provocative positions. However, use of a brace is considered one of the treatments to prevent re-dislocation after an initial dislocation, and its effectiveness is controversial [28,29]. This study evaluated the efficacy of bracing in the prevention of an initial dislocation after THA during the early postoperative period.

In this study, two patients, who were in the safe range based on a radiographic evaluation and had femoral head sizes of 22 and $26 \mathrm{~mm}$, respectively due to their acetabular cup size although we deeply recognized these sizes were considered small by today's standards to prevent hip dislocation, unfortunately experienced dislocation within 2 weeks while carelessly assuming a provocative position without wearing a hip brace despite repeated cautioning by the medical staff. No primary hip dislocation occurred during the first 6 postoperative months when a hip brace was worn. Careless assumption of a provocative position in the ADL may be one of the major factors responsible for hip dislocation in the early postoperative period after surgery, when the soft tissues have not healed sufficiently to restrict such positions. All of our patients were clearly told to avoid excessive rotation that could jeopardize soft-tissue and abductor repairs during the brace-wearing period. Our results suggest that a hip brace helps patients recognize the provocative position and accelerates soft tissue healing without attenua tion and detachment.

Post-discharge costs are an important issue in arthroplasty surgery. Lavernia et al. [30] reported that the total costs were significantly lower in patients discharged home directly vs those sent to a comprehensive rehabilitation unit who subsequently received home care (\$2405 vs $\$ 13,435, P<0.001)$. Peak et al. [23] reported that there was an additional expenditure of approximately \$655 in the restricted group for an abduction pillow, elevated toilet seat, and elevated chair per THA patient. As the Medicare reimbursement for a hip abduction brace is approximately $\$ 875$, the Medicaid reimbursement is approximately $\$ 750$ [29], and our brace costs approximately $\$ 800$, discharge directly to home with a hip brace to prevent initial dislocation may be a reasonable choice for 
reducing the post-discharge costs after THA with shorter contemporary in-hospital stay. In addition, Woolson and Rahimtoola [14] recommended the prophylactic use of a modified hip spica cast or hip brace during the first few weeks after surgery to prevent dislocation, especially for elderly patients with a history of alcoholism, senility, or confusional states. The abduction brace may play a very important role for elderly patients with such cerebral dysfunction and restrict the adoption of provocative postures, saving the additional cost of reducing their dislocations.

In conclusion, the abduction hip brace allows the patient to exercise while limiting only the direction of adduction and controlling the other directions as deemed necessary by the surgeons. Although the numbers in this study were so small that we cannot draw definitive conclusions, applying a brace during the early postoperative period may be effective and benefit the patients, especially given the current trend to perform THA as day surgery, because patients with braces are less likely to assume provocative postures after THA without any other devices such as a high toilet seat, use of a reacher or grabber, an abduction pillow, and a high chair etc.

\section{REFERENCES}

[1] M. A. Ali Khan, P. H. Brakenbury and I. S. Reynolds, “Dislocation Following Total Hip Replacement," Journal of Bone and Joint Surgery, Vol. 63, No. 2, 1981, pp. 214218

[2] D. J. Berry, M. von Knoch, C. D. Schleck and W. S. Harmsen, "The Cumulative Long-Term Risk of Dislocation after Primary Charnley Total Hip Arthroplasty,” Journal of Bone and Joint Surgery, Vol. 86, No. 1, 2004, pp. 9-14.

[3] J. J. Callaghan, B. E. Heithoff, D. D. Goetz, et al., "Prevention of Dislocation after Hip Arthroplasty. Lessons from Long-Term Follow-Up,” Clinical Orthopaedics and Related Research, Vol. 393, 2001, pp. 157-162. doi:10.1097/00003086-200112000-00018

[4] T. K. Cobb, B. F. Morrey and D. M. Ilstrup, "The Elevated-Rim Acetabular Liner in Total Hip Arthroplasty: Relationship to Postoperative Dislocation,” Journal of Bone and Joint Surgery, Vol. 78, No. 1, 1996, pp. 80-86.

[5] H. A. Demos, C. H. Rorabeck, R. B. Bourne, S. J. MacDonald and R. W. McCalden, "Instability in Primary Total Hip Arthroplasty with the Direct Lateral Approach,” Clinical Orthopaedics and Related Research, Vol. 393, 2001, pp. 168-180. doi:10.1097/00003086-200112000-00020

[6] U. Hedlundh, L. Ahnfelt, C. H. Hybbinette, J. Weckström and H. Fredin, "Surgical Experience Related to Dislocations after Total Hip Arthroplasty," Journal of Bone and Joint Surgery, Vol. 78, No. 2, 1996, pp. 206-209.

[7] R. E. White, T. J. Forness, J. K. Allman and D. W. Junick, "Effect of Posterior Capsular Repair on Early Dislocation in Primary Total Hip Replacement,” Clinical Orthopaedics and Related Research, Vol. 393, 2001, pp. 163-167. doi:10.1097/00003086-200112000-00019

[8] J. F. Williams, M. J. Gottesman and T. H. Mallory, "Dislocation after Total Hip Arthroplasty. Treatment with an Above Knee Spica Cast," Clinical Orthopaedics and Related Research, Vol. 171, 1982, pp. 53-58.

[9] R. Y. Woo and B. F. Morrey, "Dislocations after Total Hip Arthroplasty,” Journal of Bone and Joint Surgery, Vol. 64, No. 9, 1982, pp. 1295-1306.

[10] M. Khatod, T. Barber, E. Paxton, R. Namba and D. Fithian, "An Analysis of the Risk of Hip Dislocation with a Contemporary Total Joint Registry,” Clinical Orthopaedics and Related Research, Vol. 447, 2006, pp. 19-23. doi:10.1097/01.blo.0000218752.22613.78

[11] R. Biedermann, A. Tonin, M. Krismer, et al., "Reducing the Risk of Dislocation after Total Hip Arthroplasty. The Effect of Orientation of the Acetabular Component," Journal of Bone and Joint Surgery, Vol. 87, No. 6, 2005, pp. 762-769. doi:10.1302/0301-620X.87B6.14745

[12] C. B. Phillips, J. A. Barrett, E. Losina, et al., "Incidence Rates of Dislocation, Pulmonary Embolism, and Deep Infection during the First Six Months after Elective Total Hip Replacement," Journal of Bone and Joint Surgery, Vol. 85, No. 1, 2003, pp. 20-26.

[13] C. D. Fackler and R. Poss, "Dislocation in Total Hip Arthroplasties,” Clinical Orthopaedics and Related Research, Vol. 151, 1980, pp. 169-178.

[14] S. T. Woolson and Z. O. Rahimtoola, "Risk Factors for Dislocation during the First 3 Months after Primary Total Hip Replacement,” Journal of Arthroplasty, Vol. 14, No. 6, 1999, pp. 662-668. doi:10.1016/S0883-5403(99)90219-X

[15] J. L. Masonis and R. B. Bourne, "Surgical Approach, Abductor Function, and Total Hip Arthroplasty Dislocation," Clinical Orthopaedics and Related Research, Vol. 405, 2002, pp. 46-53. doi:10.1097/00003086-200212000-00006

[16] L. D. Dorr, A. W. Wolf, R. Chandler and J. P. Conaty, "Classification and Treatment of Dislocations after Total Hip Arthroplasty,” Clinical Orthopaedics and Related Research, Vol. 173, No. 173, 1983, pp. 151-158.

[17] B. M. Jolles, P. Zangger and P. F. Leyvraz, "Factors Predisposing to Dislocation after Primary Total Hip Arthroplasty,” Journal of Arthroplasty, Vol. 17, No. 3, 2002, pp. 282-288. doi:10.1054/arth.2002.30286

[18] G. E. Lewinnek, J. L. Lewis, R. Tarr, C. L. Compere and J. R. Zimmerman, "Dislocations after Total hip- Replacement Arthroplasty," Journal of Bone and Joint Surgery, Vol. 60, No. 2, 1978, pp. 217-220.

[19] M. Soong, H. E. Rubash and W. Macaulay, "Dislocation after Total Hip Arthroplasty," Journal of the American Academy of Orthopaedic Surgeons, Vol. 12, No. 5, 2004, pp. 314-321.

[20] D. J. Berry, M. von Knoch, C. D. Schleck and W. S. Harmsen, "Effect of Femoral Head Diameter and Operative Approach on Risk of Dislocation after Primary Total Hip Arthroplasty,” Journal of Bone and Joint Surgery, Vol. 87, No. 11, 2005, pp. 2456-2463. 


\section{doi:10.2106/JBJS.D.02860}

[21] P. L. Kung and M. D. Ries, "Effect of Femoral Head Size and Abductors on Dislocation after Revision THA," Clinical Orthopaedics and Related Research, Vol. 465, 2007, pp. 170-174.

[22] R. A. Berger, "Total Hip Arthroplasty Using the Minimally Invasive Two-Incision Approach,” Clinical Orthopaedics and Related Research, Vol. 417, 2003, pp. 232241.

[23] E. L. Peak, J. Parvizi, M. Ciminiello, et al., "The Role of Patient Restrictions in Reducing the Prevalence of Early Dislocation Following Total Hip Arthroplasty, A Randomized, Prospective Study," Journal of Bone and Joint Surgery, Vol. 87, No. 2, 2005, pp. 247-253. doi:10.2106/JBJS.C. 01513

[24] D. W. Murray, "The Definition and Measurement of Acetabular Orientation," Journal of Bone and Joint Surgery, Vol. 75, No. 2, 1993, pp. 228-232.

[25] W. M. Goldstein, T. F. Gleason, M. Kopplin and J. J. Branson, "Prevalence of Dislocation after Total Hip Arthroplasty through a Posterolateral Approach with Partial Capsulotomy and Capsulorrhaphy,” Journal of Bone and Joint Surgery, Vol. 83, Suppl. 2, 2001, pp. 2-7.
[26] P. M. Pellicci, M. Bostrom and R. Poss, "Posterior Approach to Total Hip Replacement Using Enhanced Posterior Soft Tissue Repair,” Clinical Orthopaedics and Related Research, Vol. 355, 1998, pp. 224-228. doi:10.1097/00003086-199810000-00023

[27] T. Youm, S. G. Maurer and S. A. Stuchin, "Postoperative Management after Total Hip and Knee Arthroplasty," Journal of Arthroplasty, Vol. 20, No. 3, 2005, pp. 322-324. doi:10.1016/j.arth.2004.04.015

[28] M. L. Clayton and R. G. Thirupathi, "Dislocation Following Total Hip Arthroplasty. Management by Special Brace in Selected in Patients," Clinical Orthopaedics and Related Research, Vol. 177, 1983, pp. 154-159.

[29] H. Dewal, S. L. Maurer, P. Tsai, R. Hiebert and P. E. Di Cesare, "Efficacy of Abduction Bracing in Management of Total Hip Arthroplasty Dislocation,” Journal of Arthroplasty, Vol. 19, No. 6, 2009, pp. 733-738. doi:10.1016/j.arth.2004.02.041

[30] C. J. Lavernia, M. R. D’Apuzzo, V. H. Hernandez, D. J. Lee and M. D. Rossi, "Postdischarge Costs in Arthroplasty Surgery,” Journal of Arthroplasty, Vol. 21, No. 6, 2006, pp. 144-150. doi:10.1016/j.arth.2006.05.003 